\title{
Special issue on PODC 2018 and DISC 2018
}

\section{Hagit Attiya ${ }^{1}$}

Accepted: 6 July 2021 / Published online: 15 July 2021

(c) The Author(s), under exclusive licence to Springer-Verlag GmbH Germany, part of Springer Nature 2021

This special issue of Distributed Computing is based on papers that originally appeared as extended abstracts in the Proceedings of the 37th Symposium on Principles of distributed computing (PODC 2018), held in Egham, United Kingdom, and in the Proceedings of the 32nd International Symposium on Distributed Computing (DISC 2018), held in New Orleans, Louisiana, USA.

The papers for the Special Issue were chosen by the respective Program Committees from the regular papers presented at the symposium, based on their quality and representation of the spectrum of topics encompassed by the symposium. In addition to being reviewed, in preliminary form, by the Program Committee, the full papers submitted for the Special Issue were refereed according to the standard practices of Distributed Computing.

We thank the program committees, the handling editors, the referees and the authors of these papers.

Publisher's Note Springer Nature remains neutral with regard to jurisdictional claims in published maps and institutional affiliations.

Hagit Attiya

hagit@cs.technion.ac.il

1 Technion, Haifa, Israel 